\title{
La mamografía es menos costo-efectiva en mujeres de 40 a 49 años
}

Cost-effectiveness of extending screening mamography guidelines to include women 40 to 49 years of age. Salzmann P, Kerlikowske K, Phillips K. Ann Int Med 1997;127:955-965.

\author{
Objetivo \\ Comparar la costo-efectividad de la mamografía de rastreo \\ (screening) en mujeres de distintas edades.
}

\section{Diseño}

Análisis de costo-efectividad*.

Lugar

Estudio poblacional en EE.UU.

\section{Pacientes}

Mujeres mayores de 39 años de la población general.

\section{Intervención}

Se comparó: 1) Rastreo cada 2 años en mujeres de 50 a 69 años vs. No rastreo; 2) Rastreo cada 18 meses de los 40 a los 49 años, seguido por rastreo cada 2 años de 50 a 69 vs Rastreo cada 2 años de los 50 a 69 años.
Medición de costos y resultados principales

Expectativa de vida, costo, y costo-efectividad incremental*

\section{Resultados Principales}

El rastreo en mujeres de 50 a 69 años mejoró la expectativa de vida promedio en 12 días a un costo de $\$ 704$ por mujer; esto resulta en una tasa de costo-efectividad de $\$ 21.400$ por año de vida salvado. Si el rastreo se extiende a las mujeres de 40 a 49 años, la expectativa de vida aumenta 2.5 días más a un costo adicional de $\$ 676$ por mujer. La costo-efectividad incremental en este grupo fue de $\$ 105.000$ por año de vida salvado. Un análisis de sensibilidad reportó que hay un $75 \%$ de probabilidad de que el rastreo en el. grupo de 50 a 69 el año de vida salvado sea menor a $\$ 50.000$, mientras que esta probabilidad es del $7 \%$ en el grupo de 40 a 49 años.

\section{Conclusiones}

La mamografía de rastreo es cinco veces más costo-efectiva en mujeres de 50 a 69 años que en las de 40 a 49 años.

\section{COMENTARIO}

Existe consenso para recomendar la mamografía como método de rastreo en las mujeres de riesgo habitual de 50 a 69 años; y realizarla cada dos años es tan efectiva como hacerla en forma anual (1). En pacientes de alto riesgo (familiar de 1er grado con cáncer premenopáusico) se recomienda empezar el rastreo anual a los 35 años. Hay discrepancia con respecto a las recomendaciones en mujeres de riesgo promedio de 40 a 49 años. Las fuerzas de tareas preventivas de EE.UU., canadiense, y el reciente consenso del Instituto Nacional de Salud de EE.UU. no recomiendan el rastreo universal en este grupo; mientras que las asociaciones de oncólogos y radiólogos se inclinan a su favor. Para decidir políticas de salud es necesario contar con evidencia rigurosa acerca de los beneficios, riesgos y costos de una intervención, así como también tener en cuenta los valores de la sociedad. Una herramienta más para ayudar a tomar estas decisiones es el análisis de costo-efectividad*; un método para evaluar y comparar distintas estrategias de salud con respecto no sólo a la efectividad de una intervención, sino también cuánto le cuesta a la sociedad esa cuota extra de efectividad (2). Los resultados de estos estudios se expresan como \$/año de vida salvado; o más recientemente como \$/QALY (año de vida ajustado por calidad), para incorporar también la calidad de vida. El estudio de Salzmann y col. es una buena muestra de este tipo de análisis. A pesar de hacer el trabajo utilizando estimaciones que traten de favorecer al rastreo, concluyen que la mamografía es mucho menos costo-efectiva en mujeres de 40 a 49 años. Esto se debe principalmente a que el beneficio en esta población es pequeño y recién se observa luego de los 10-14 años del comienzo del rastreo (para prevenir una muerte habría que rastrear periódicamente 2.500 mujeres de 40-49 años; y sólo a 270 mujeres de 50-69 años). Los países desarrollados consideran costo-efectivas a estrategias con un índice cercano o menor al de $\$ 50.000$ por QALY. Tanto el resultado principal de este estudio como los análisis de sensibilidad (que confirmaron la estabilidad de las conclusiones) muestran que la mamografía en mujeres de 40-49 años es costo-inefectiva según este umbral. Esta relación costo-efectividad podría variar si se toman en cuenta los costos y la efectividad de la mamografía en países en desarrollo. En conclusión, los programas de mamografía de rastreo deben concentrarse en mujeres de 50 a 69 años. Extender el rastreo a las de 40-49 años es por ahora demasiado costoso tomando en cuenta el marginal beneficio. Es importante ser concientes que el presupuesto en salud es limitado, y lo que se gasta en una estrategia poco efectiva no puede ser utilizado en otra que depare mayor beneficio.

*Ver glosario.

Dr. Federico Augustovski

Unidad de Medicina Familiar y Preventiva Hospital Italiano de Buenos Aires 